\title{
Abrupt climate progress
}

\author{
The Earth's climate is a complex system and palaeoclimate reconstructions can be used to test and expand on the \\ knowledge gained from physical models during intervals of rapid climate fluctuations.
}

A dvances in understanding the physical basis of climate change have recently been highlighted by the awarding of the Nobel Prize in Physics to two climate modellers, Syukuro Manabe and Klaus Hasselmann, and theoretical physicist, Giorgio Parisi. A Collection of their work and related studies from across the Nature Portfolio shows how and why Earth's climate can vary abruptly - sometimes causing disruptions detrimental to societies around the globe who rely on stable, or at least predictable, temperature and precipitation conditions. This work underpins projections of climate change in coming decades, in part by helping to clarify the importance and causes of random as well as more systemic shifts.

The theoretical understanding of climate variability can be tested on palaeoclimate reconstructions that extend back beyond the instrumental record, which are especially useful when they can resolve changes that take place over decades to centuries. These records can also help fill gaps in understanding climate variability - some of which may be relevant for future climate - over long intervals (tens to hundreds of thousands of years) that are computationally challenging to simulate at high time or spatial resolution.

Reconstruction of past climate states requires accurate and precise palaeontological and geochemical tools that can constrain past environmental conditions at a temporal and spatial resolution relevant to the question at hand. For example, a thousand-year-long record of annual temperature changes could be used to test for the influence of past $\mathrm{El}$ Niño-like events (typically every 2-7 years), but it won't reveal much about shifting seasonal temperature changes or events that only occur every few thousand years. With appropriate experimental design, reconstructions can show how quickly the climate system can respond to perturbations and whether recent climate events are one-offs or part of repeated, and potentially predictable, patterns.

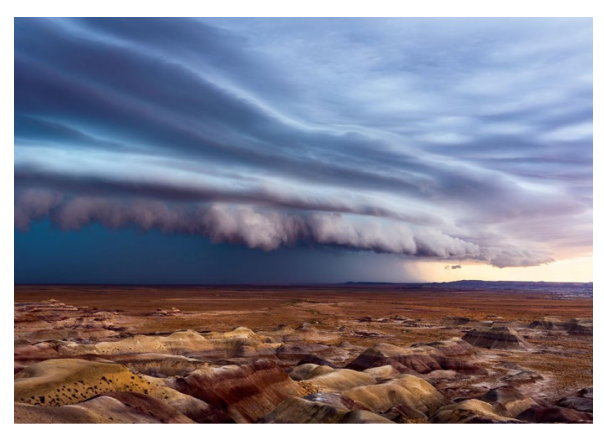

Credit: John Sirlin / Alamy Stock Photo

The transition towards warmer conditions following the Last Glacial Maximum at the tail end of the Pleistocene (from about $19 \mathrm{ka}$ to $11 \mathrm{ka}$ ) is an especially useful time interval for understanding the occurrence and physical cause of rapid climate variability. This interval was punctuated by a series of short-term climate perturbations - generally global to regional warm or cold reversals lasting hundreds to thousands of years that began and ended abruptly. While substantial uncertainties as to what drives these shifts remain, a Review by Brovkin et al. shows that gradual disruption of one climate subsystem can suddenly propagate to another (Nat. Geosci. 14, 550-558; 2021). For example, as North American glaciers melt they induce rapid disruption in North Atlantic circulation, resulting in quickly cascading changes with complex spatial effects like rainfall patterns over Europe. While the boundary conditions of the last glacial period mean that these events are not directly analogous to our warmer future, this work does help to build our understanding of the processes that control the magnitude and rates of climatic change.

Rapid climate perturbations likely accompanied earlier Pleistocene glacial periods, but these become harder to resolve given increasingly spotty availability of suitable proxy records. In this issue, an Article by Sun et al. pushes back understanding of abrupt climate variability by precisely reconciling four 800,000 year-long centennial-scale elemental proxy records - sensitive to local temperature and hydroclimate conditions - spanning the Northern Hemisphere. The authors find coherent, millennial-scale climate variations regularly occurred throughout the study interval. The frequency and amplitude of this variability was controlled by both the presence of ice sheets as well as the influence of insolation changes associated with periodic variations in Earth's orbit.

Building on observations of rapid climate variability through the Pleistocene, an accompanying Article by Zhang et al. uses climate models to investigate how the small changes in solar energy reaching the Earth's surface associated with orbital changes can induce millennial-scale variability. The authors show that subtle insolation gradients between high and low latitudes under particular orbital conditions can induce oscillations consistent with the millennial-scale variability observed in Sun et al.

The combined results of the articles cited here show the value, as well as the challenges, of extending high-resolution climate work further back in time through a wider swath of Pleistocene glaciations - all with unique global temperature and atmospheric $\mathrm{CO}_{2}$ background conditions. Atmospheric $\mathrm{CO}_{2}$ levels are continuing to rise well beyond the range during the Pleistocene glacial cycles, and figuring out the capacity of the Earth system to quickly adjust to this new reality is becoming ever more important. Palaeoclimate records can, when of sufficient resolution and when collected in the right place, contextualize some of the hidden climatic machinery revealed by the work of this year's Nobel Prize winners and many others - that is likely to dictate the societal consequences of these events: we just need the right tools to pull back the curtain.

Published online: 3 November 2021 https://doi.org/10.1038/s41561-021-00854-6 